\title{
Free testosterone and cardiometabolic parameters in men: comparison of algorithms
}

\author{
Stine A Holmboe ${ }^{1,2}$, Ravi Jasuja ${ }^{3}$, Brian Lawney³, Lærke Priskorn ${ }^{1,2}$, Niels Joergensen ${ }^{1,2}$, Allan Linneberg ${ }^{4,5}$, \\ Tina Kold Jensen ${ }^{1,2,6}$, Niels Erik Skakkebæk ${ }^{1,2}$, Anders Juul ${ }^{1,2}$ and Anna-Maria Andersson ${ }^{1,2}$ \\ 1Department of Growth and Reproduction, Rigshospitalet, University of Copenhagen, Blegdamsvej, Copenhagen, Denmark \\ ${ }^{2}$ The International Research Centre in Endocrine Disruption of Male Reproduction and Child Health (EDMaRC), Rigshospitalet, University of Copenhagen, \\ Copenhagen, Denmark \\ ${ }^{3}$ Research Program in Men's Health: Aging and Metabolism, Brigham and Womens Hospital, Harvard Medical School, Boston, Massachusetts, USA \\ ${ }^{4}$ Centre for Clinical Research and Prevention, Frederiksberg Hospital, Copenhagen, Denmark \\ ${ }^{5}$ Department of Clinical Medicine, Faculty of Health and Medical Sciences, University of Copenhagen, Copenhagen, Denmark \\ ${ }^{6}$ Department of Environmental Medicine, Institute of Public Health, University of Southern Denmark, Odense, Denmark
}

Correspondence should be addressed to A-M Andersson: anna@rh.dk

\begin{abstract}
Objective: Calculating the free testosterone level has gained increasing interest and different indirect algorithms have been suggested. The objective was to compare free androgen index (FAl), free testosterone estimated using the linear binding model (Vermeulen: (FTV) and the binding framework accounting for allosterically coupled SHBG monomers (Zakharov: cFTZ) in relation to cardiometabolic conditions.

Design: A prospective cohort study including 5350 men, aged 30-70 years, participating in population-based surveys (MONICA I-III and Inter99) from 1982 to 2001 and followed until December 2012 with baseline and follow-up information on cardiometabolic parameters and vital status.

Results: Using age-standardized hormone levels, FAl was higher among men with baseline cardiometabolic conditions, whereas CFTV and cFTZ levels were lower compared to men without these conditions as also seen for total testosterone. Men in highest quartiles of cFTV or CFTZ had lower risk of developing type 2 diabetes (cFTV: HR $=0.74$ $(0.49-1.10)$, cFTZ: $H R=0.59(0.39-0.91))$ than men in lowest quartile. In contrast, men with highest levels of FAl had a 74\% (1.17-2.59) increased risk of developing type 2 diabetes compared to men in lowest quartile. Conclusion: The association of estimated free testosterone and the studied outcomes differ depending on algorithm used. cFTV and cFTZ showed similar associations to baseline and long-term cardiometabolic parameters. In contrast, an empiric ratio, FAl, showed opposite associations to several of the examined parameters and may reflect limited clinical utility.
\end{abstract}
Key Words
- free testosterone
- total testosterone
- metabolic syndrome
- follow-up study

\section{Introduction}

Testosterone in serum is primarily bound to proteins with only a minor fraction circulating as free unbound testosterone. The major high-affinity binding protein is sex hormone-binding globulin (SHBG) (1). Because of the short half-life of unbound testosterone in serum and the hypothalamic adjustment of testosterone, changes in SHBG levels will strongly influence the level of total testosterone.

According to the free hormone hypothesis, the free testosterone and the fraction of testosterone loosely bound to albumin are considered the fractions that are bioavailable for the target tissues (2). The testosterone
Endocrine Connections (2021) 10, 220-229 
level is affected by health status and different lifestyle factors and in general, there is agreement between the free and total levels of testosterone regarding the direction and magnitude of the observed effects $(3,4)$. However, some studies including both total and free testosterone have indicated that free testosterone rather than total testosterone (TT) is associated with androgen deficiencyrelated symptoms $(5,6)$. Consequently, determining the free or bioavailable testosterone level has gained increasing interest over the years (7). Equilibrium dialysis is considered the gold standard method for measuring free testosterone but is a time-consuming method mostly used for research purposes and not widely available (1, 8). Therefore, alternative indirect equations for the estimation of free testosterone have been developed. Free androgen index (FAI) is probably the simplest of these equations as it is calculated as the ratio between TT and SHBG $($ TT $\times 100 /$ SHBG). As indicated by the name, FAI is not an estimate of the free testosterone concentration but simply an index of the balance between serum testosterone and SHBG. While it is recognized that FAI is not based on any biophysical characterization, it continues to be used by investigators $(9,10)$. Several algorithms based on law-of-mass-action equations requiring information on the level of TT and SHBG have been developed with varying degree of correlation with equilibrium dialysis and some have been suggested as reasonable alternatives to direct measurements (1). One of the most commonly used indirect algorithms, which have been acknowledged by the Endocrine Society because of its clinical relevance in relation to hypogonadism, was suggested by Vermeulen et al. (1, 11, 12). The algorithm by Vermeulen is constructed using linear model of binding where monomers within the SHBG dimer were assumed to have identical affinity (11). This model predates the resolution of crystal structure of dimeric SHBG (13). Subsequently, several detailed biophysical studies have found the SHBG binding with testosterone to be a non-linear, multiphasic process (14). More recently, Zakharov et al. demonstrated that SHBG monomers within the dimer are allosterically coupled. Allostery in the multimeric, heteromeric or multidomain proteins allows for fine-tuned binding and release dynamics of ligands in diverse systems spanning oxygen transport by hemoglobin to transcription factors signaling. Since testosterone concentrations change several orders of magnitude during development, puberty, pregnancy and aging, it is conceivable that the SHBG dimer was designed to act as a non-linear logarithmic sensor (15). If the two monomers were identical in the dimer, as is assumed by the simplified linear model, there will be no evolutionary benefit for dimeric structure of SHBG. Detailed biophysical characterization of SHBG/testosterone interaction led to the development of multistep, non-linear binding model upon the ensemble allostery framework (16), which also has been validated against equilibrium dialysis (17).

Other studies have attempted to compare free testosterone levels measured directly by equilibrium dialysis with indirect estimations of free testosterone (11, $17,18)$. However, the predictive value of the different free testosterone estimations in relation to androgen deficiency parameters was not investigated and remains unknown. Numerous studies have shown that lower levels of TT and SHBG are associated with risk factors for cardiovascular disease such as obesity and hyperglycemia although the causal mechanism behind this is unclear, reviewed in (19). Also lower level of free testosterone estimated by Vermeulen et al. has been associated with risk factors for cardiovascular disease (19). However, free testosterone calculated using different algorithms and the association to cardiometabolic parameters have not been compared before.

Thus, the aim in the following was twofold; first we examined the baseline association between the different free testosterone algorithms and cardiometabolic conditions and secondly, the aim was to describe the predictive value of the different algorithms of free testosterone in relation to the long-term development of type 2 diabetes, cardiovascular disease and mortality. To elucidate this, a cohort consisting of more than 5000 adult men from the general population with hormone levels available as well as information on baseline and follow-up information on cardiometabolic parameters was used.

\section{Materials and methods}

\section{Study population}

The study comprised serum samples from 5350 randomly selected Danish men participating in four cross-sectional surveys at Research Centre for Prevention and Health at Glostrup University Hospital as described previously (20). The four surveys MONICA I, MONICA II, MONICA III and INTER99 were conducted in 1982, 1987, 1992 and between 1999 and 2001, respectively $(21,22)$. All participants from the four surveys were drawn as random samples of 30-, 40-, 50-, 60-, and 70-year-old men from the Danish Civil Registration System in which all Danish inhabitants are

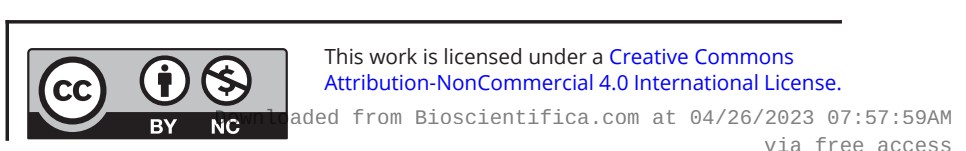


registered. The participation rates in the four surveys were $81,75,69$, and $51 \%$, respectively.

All participants went through a physical examination and filled out a detailed questionnaire on lifestyle factors and health status and had a blood sample drawn while fasting (23). Serum samples were analyzed for lipids and stored in aliquots at $-18^{\circ} \mathrm{C}$ until further analyses. The study was approved by the Ethical Committee of the County of Copenhagen, and all participants had provided informed consent.

\section{Hormone analyses and calculations}

Total Testosterone (TT) and SHBG were measured by time-resolved fluoroimmunoassay (DELFIA; Wallac Oy, Turku, Finland) and time-resolved immunofluorometric assay (DELFIA), respectively, as described previously (20, $24)$. The intra- and interassay coefficients of variation were less than $12 \%$ for testosterone and less than $8 \%$ for SHBG. Hormone measurements were performed in the same laboratory in 2004. Because of varying storage periods (3-22 years) and varying number of thawings, the hormone levels were initially corrected for evaporation according to median serum $\mathrm{Na}^{+}$-levels measured in samples from each of the four surveys as previously validated (24). Indirect estimations of free testosterone were calculated in three different ways; the free androgen index (FAI) was calculated as the ratio between measured levels of testosterone and SHBG times 100 (testosterone $\times$ 100/SHBG), and furthermore, free testosterone was calculated based on levels of TT and SHBG according to the linear approach by Vermeulen (cFTV) (11) and the framework accounting for ensemble allostery in SHBG dimers allowing non-linear association between testosterone and SHBG (cFTZ) (17).

\section{Follow-up information}

In Denmark, all residents have a unique ten-digit identification number, the so-called Civil Personal Register (CPR) number, allowing linkage of data from virtually complete registries on an individual level (25). Thus, follow-up information from national registries was obtained based on the CPR number from the participants. The participants were linked to the Danish Civil Registration System in which information on vital status and migration was available and followed until December 17,2012 . To obtain information on cardiovascular disease (CVD)-specific mortality the CPR number was furthermore linked to the Danish Register of Causes of Death (26).
Finally, selected information on hospitalizations was obtained from the Danish National Patient Register in which information on all hospital admissions since 1977 has been registered until the end of follow-up (December 31, 2011). Specifically, information on type 2 diabetes (T2D) and ischemic heart disease (IHD) was obtained defined according to the International Classification of Diseases (ICD) as: ICD-8: 250/ICD-10: E11 and ICD-8: 410-414/ICD-10: I20-I25, respectively. Four men were excluded because of a loss of follow-up. Follow-up time for the remaining participants was calculated as the time from their baseline examination until the time of event or end of follow-up, whichever occurred first. Median (25th-75th percentile) follow-up time was 19.8 years (12.0-25.3 years). Furthermore, subjects $(n=23)$ were excluded from the analyses if they had a prior diagnosis of impaired testis function leaving 5323 men for analyses of long-term outcomes $(20,27)$. Participants who emigrated during the follow-up period $(n=62)$ contributed with person time at risk until date of emigration, after which they were censored.

\section{Statistical methods}

Mean, S.D. and relative S.D. (sample S.D. $\sigma /$ sample mean $\mu \times 100)$ were calculated for each of the three free testosterone variables. Initially, cFTV and cFTZ levels were visually compared by use of scatter plot and Bland-Altman plot illustrating mean free testosterone levels in relation to the absolute difference between the two calculated variables. To allow direct comparison of the algorithms (independent of the differences in absolute values) agestandardized z-scores were calculated as previously described (20). Differences in mean absolute hormone levels as well as mean z-score levels of FAI, cFTV, cFTZ, TT and SHBG were stratified according to baseline lifestyle and health-related characteristics and tested using general linear regression models.

To compare the predictive value of the three free testosterone algorithms in relation to cardiometabolic conditions, Cox proportional hazards models with age as the underlying time scale were used to evaluate the association between hormone z-scores and T2D, IHD, CVD mortality and all-cause mortality. Hormone z-scores were analyzed in quartiles to accommodate potential nonlinear associations and, a trend test was used to evaluate any linear trend by inserting the quartiles in the model as a continuous variable. In fully adjusted models, individuals with a missing value of at least one of the included covariates (124 individuals (2.4\%)) were excluded and

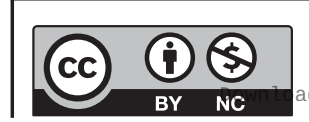

This work is licensed under a Creative Commons Attribution-NonCommercial 4.0 International License. ded from Bioscientifica.com at 04/26/2023 07:57:59AM 
quartiles recalculated. Choice of covariates was based on prior literature and all outcomes were adjusted for the same set of covariates to ensure comparability. First in a restricted model, the association between hormone z-scores and outcomes were evaluated stratified according to smoking status allowing different baseline hazards for smokers and non-smokers because of the significant impact of smoking on hormones as well as survival outcomes. Secondly, the association was furthermore adjusted for study period (1982, 1987, 1992, and 2000), BMI (<20.0, 20.0-24.99, 25.0-29.99, $\left.\geq 30 \mathrm{~kg} / \mathrm{m}^{2}\right)$, alcohol intake $(0,1-14,>14$ units/week), and exercise level (sedentary, light/moderate/vigorous). Prior to the Cox regression analysis with type 2 diabetes as the outcome, men with known type 2 diabetes at baseline were excluded $(n=163)$. Likewise, in models with IHD or CVD mortality as the outcome, men with known CVD at baseline were excluded ( $n=145)$ prior to analyses. All Cox models were checked for the assumption of proportional hazards. Hazard ratios for the three free testosterone algorithms were visually presented and compared to the estimates for TT previously published in Holmboe et al. 2015. In all analyses, $P$-values $<0.05$ were considered statistically significant. All statistical analyses were performed using $\mathrm{R}$ version 3.4.1 and IBM SPSS Statistics 22 .

\section{Results}

In general, levels of free $\mathrm{T}$ determined using the linear model systematically underestimated those determined using the ensemble allostery model (mean (s.D.) $\mathrm{cFTZ}=647.1 \quad(253.8) \mathrm{pmol} / \mathrm{L}$ and $\mathrm{cFTV}=434.5$ (163.8) $\mathrm{pmol} / \mathrm{L}$ ). The mean level of FAI, which is not directly comparable to the two other algorithms, was 67.9 (28.3). The relative standard deviations of the three calculated variables were 39,37 and $41 \%$ for cFTZ, cFTV and FAI respectively, indicating relatively similar dispersion of the frequency distribution. There was an apparent correlation between cFTV and cFTZ $(r=0.9, P<0.01)$ although the agreement decreased with higher mean free testosterone level (Fig. 1A and B) and the values from the two methods could not be interconverted by a simple scalar multiple. Stratification according to quartiles of TT indicated that higher levels of TT contributed to the difference between the two free testosterone algorithms.

Table 1 shows mean absolute hormone levels as well as age-standardized hormone levels stratified according to baseline characteristics. When stratifying according to BMI, higher BMI was associated with lower absolute levels of all hormones. In accordance, age-standardized hormone levels were lower with higher BMI except for age-standardized levels of FAI which were higher with higher BMI. Likewise, men with waist circumference $\geq 94 \mathrm{~cm}$ had lower age-standardized free testosterone levels according to Vermeulen and Zakharov but higher age-standardized FAI levels compared to men with waist circumference $<94 \mathrm{~cm} \quad(\mathrm{cFTV}=-0.20, \quad \mathrm{cFTZ}=-0.33$, and $\mathrm{FAI}=0.12$, respectively). Similarly, when stratifying according to other cardiometabolic parameters such as hypertension, elevated triglyceride level and the presence
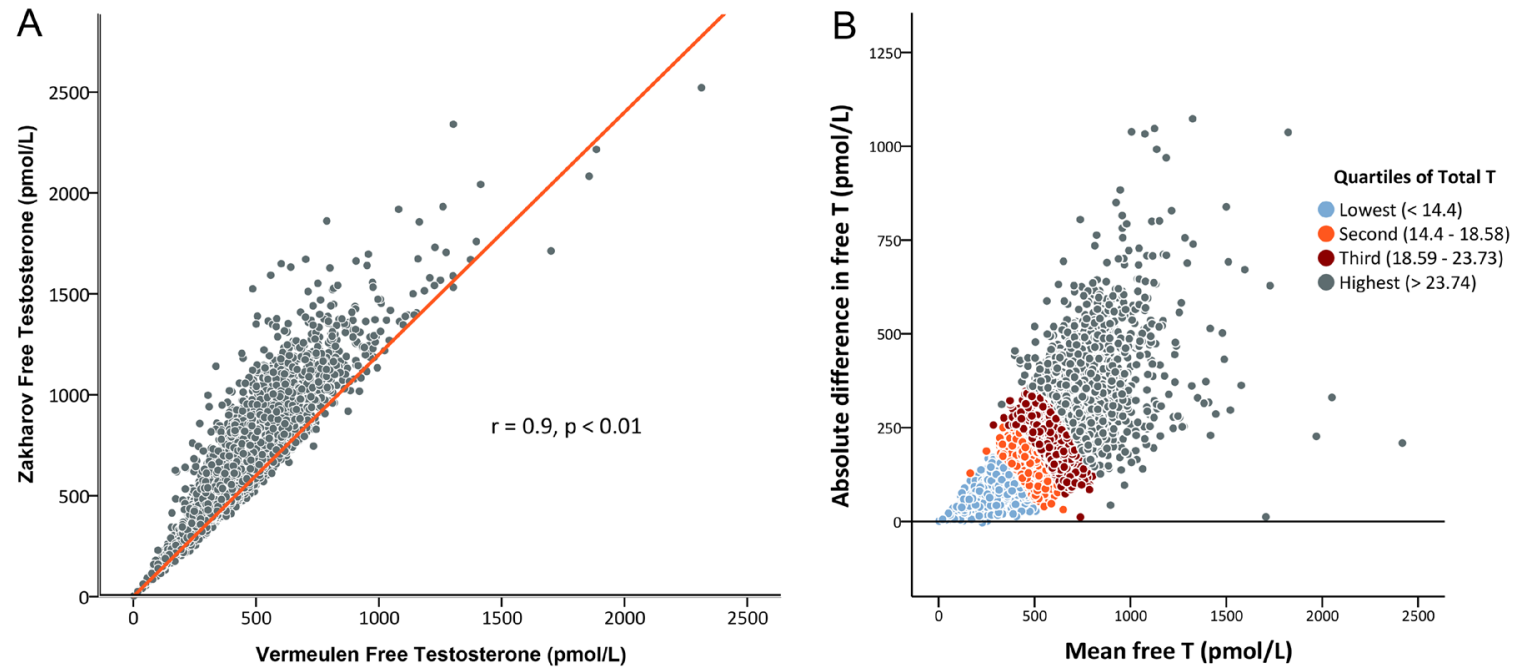

Figure 1

(A) Free testosterone according to Vermeulen equation plotted against the level according to Zakharov equation (Pearson coefficient $r=0.9, P<0.01$ ). The line represents the identity line. (B) Bland-Altman plot of the absolute difference between free testosterone calculated by Zakharov and Vermeulen equation (CFTZ - CFTV) according to mean levels of free testosterone calculated by the two equations.

https://ec.bioscientifica.com https://doi.org/10.1530/EC-20-0552 (c) 2021 The authors Published by Bioscientifica Ltd

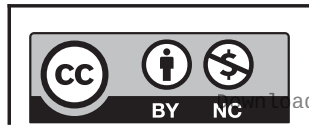

This work is licensed under a Creative Commons Attribution-NonCommercial 4.0 International License. ded from Bioscientifica.com at 04/26/2023 07:57:59AM via free access 


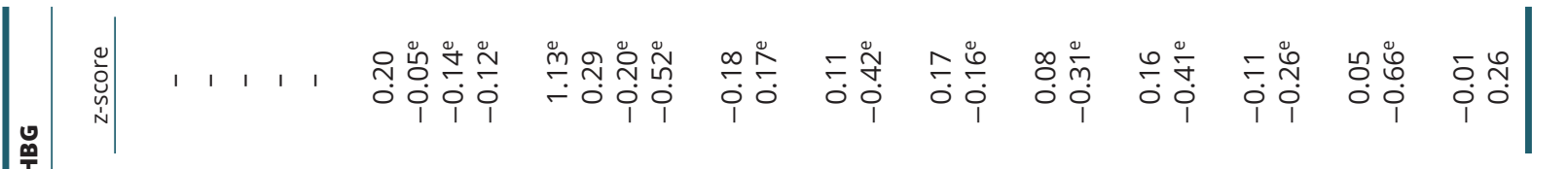

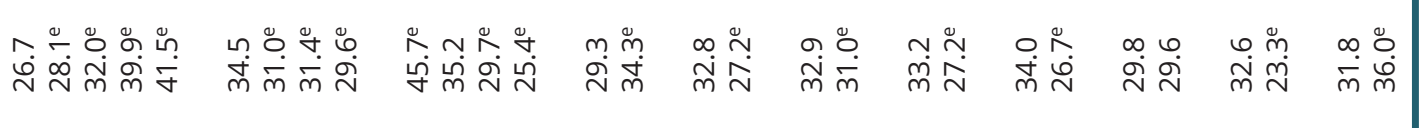

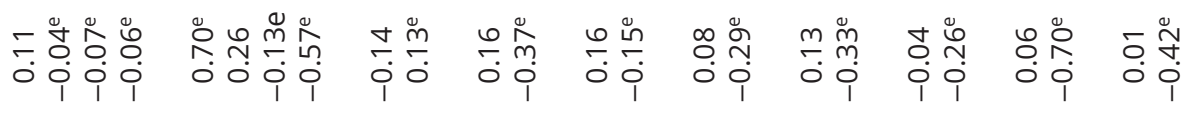

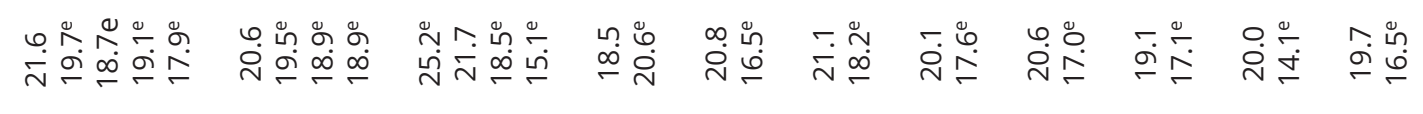

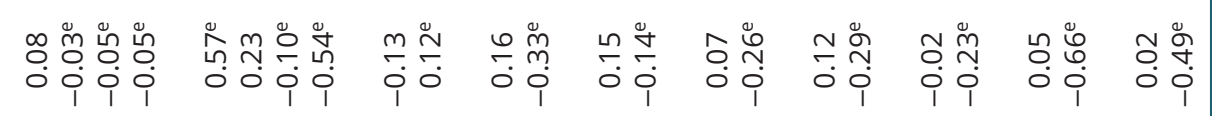

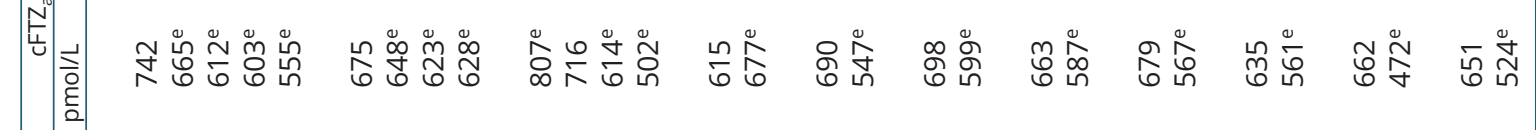

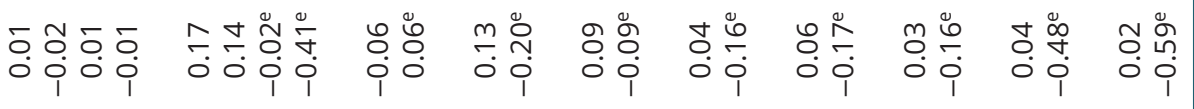

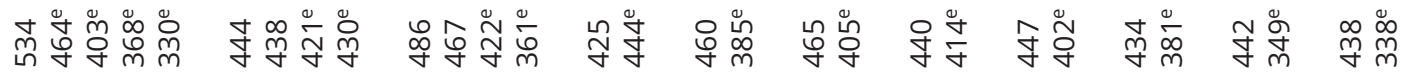

유ㅇㅠㅠ

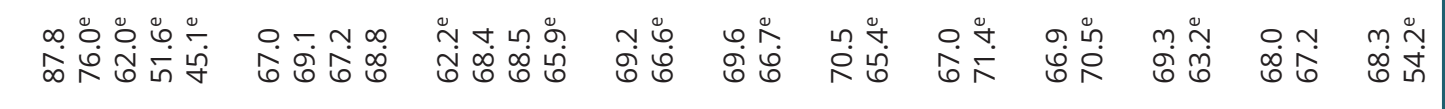

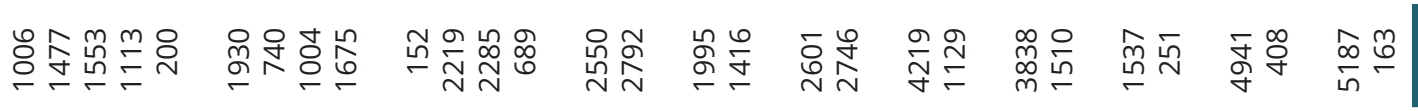


of metabolic syndrome, age-standardized levels of FAI were highest among men with these conditions compared to men without such conditions whereas age-standardized levels of cFTV and cFTZ were lowest among men with the conditions. In general, trends for cFTV and cFTZ pointed in the same direction but with more pronounced estimates for the Zakharov algorithm. Men with diabetes at baseline were characterized by significantly lower levels of age-standardized free testosterone irrespective of algorithm $(\mathrm{FAI}=-0.48, P<0.01$; cFTV $=-0.59, P<0.01$; $\mathrm{cFTZ}=-0.49, P<0.01)$.

In Cox regression analyses, quartiles of agestandardized free testosterone levels were analyzed in relation to survival outcomes (Fig. 2 and Supplementary Tables 1, 2, see section on supplementary materials given at the end of this article). Cox regression analyses revealed that higher age-standardized levels of cFTV and CFTZ were associated with lower risk of subsequent type 2 diabetes in accordance with the trend seen for TT, however only significant for cFTZ and TT $(P<0.01)$. In contrast, a significant positive association between agestandardized FAI and the risk of type 2 diabetes was seen. Thus, men in the highest quartile had a $74 \%$ increased risk of developing type 2 diabetes compared to men in the lowest quartile ( $\mathrm{HR}=1.74,95 \% \mathrm{CI}$ : 1.17-2.59). In analyses with IHD as the outcome, the three algorithms pointed in the same direction, in line with the trends seen for TT, with higher levels associated with higher risk of IHD with the most pronounced effect seen for highest quartiles of FAI $(\mathrm{FAI}, \mathrm{HR}=1.37,95 \% \mathrm{CI}=1.10-1.70 ; \mathrm{cFTV}, \mathrm{HR}=1.25$, 95\% CI=0.99-1.57: $\mathrm{cFTZ}, \mathrm{HR}=1.22,95 \% \mathrm{CI}=0.97-1.53)$.
Also, in analyses with CVD mortality as the outcome, the three algorithms followed the same trend. Thus, a lower CVD mortality risk was seen for men with agestandardized free testosterone levels in the third and fourth quartile with the most pronounced effects seen for cFTZ. In contrast, the three algorithms differed in relation to all-cause mortality analyses; no association was seen for differences in total testosterone and free testosterone estimated using the Zakharov algorithm, whereas a trend toward lower mortality risk with higher levels of FAI and cFTV was seen.

\section{Discussion}

In a cohort of more than 5000 adult men, we observed that absolute levels of free testosterone differed according to which algorithm was applied; levels estimated using the Zakharov algorithm were in general higher compared to levels using the Vermeulen algorithm. Despite differences in absolute levels, free testosterone estimated by Vermeulen and Zakharov correlated and showed similar associations to lifestyle and cardiovascular parameters. In contrast, FAI showed opposite associations to several of the examined lifestyle and cardiovascular parameters.

A limited number of studies have investigated the differences in calculations of free testosterone especially with inclusion of the algorithm suggested by Zakharov. In accordance with others, we observed that absolute levels of free testosterone estimated by Zakharov et al. tended to be higher compared to free testosterone estimated by
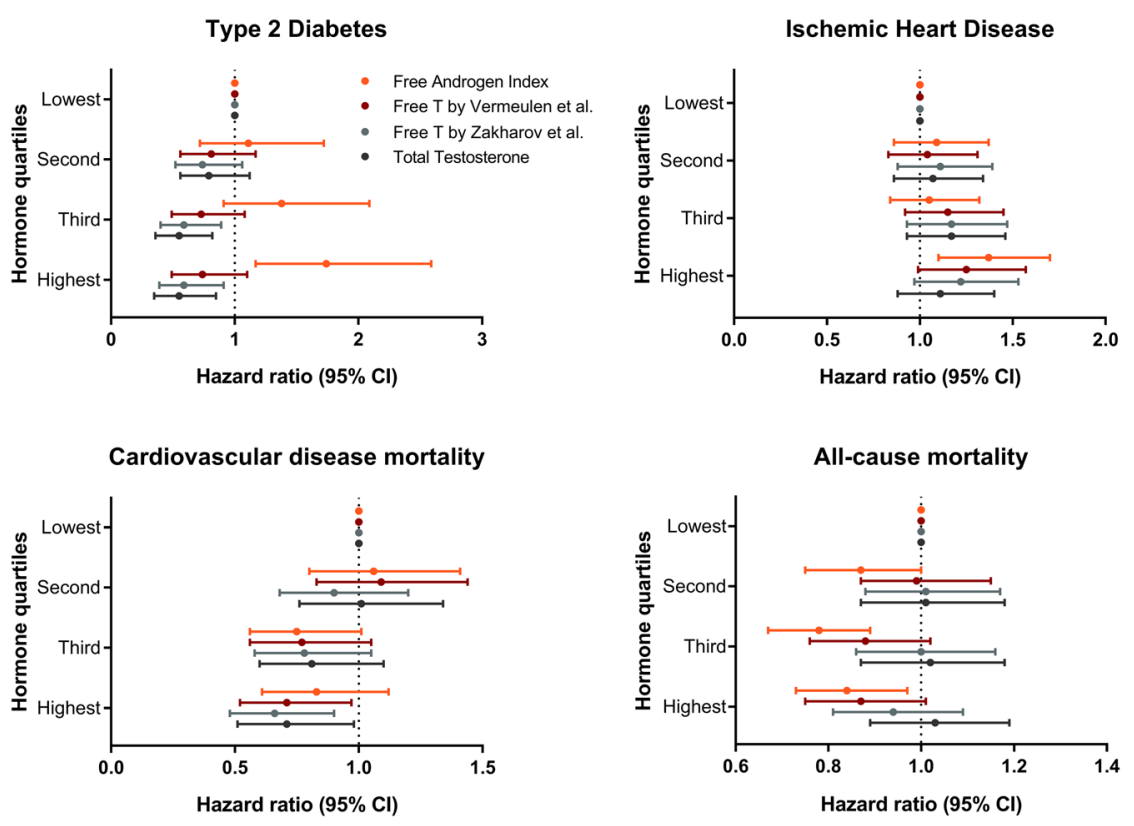

\section{Figure 2}

Hazard ratios and $95 \% \mathrm{Cls}$ for quartiles of free testosterone stratified according to algorithm (FAl, Vermeulen or Zakharov) in relation to type 2 diabetes, ischemic heart disease, cardiovascular disease mortality and all-cause mortality. All models are stratified according to smoking status and adjusted for study, BMI, alcohol consumption and physical exercise with age as the underlying time scale. 
Vermeulen et al. $(17,18)$. Because FAI is calculated as an index a direct comparison between absolute levels of FAI and the two calculated algorithms is not relevant.

To our knowledge, free testosterone calculated using different algorithms and the association with cardiometabolic parameters have not been compared before. Interestingly, in the present study, we found that men characterized by cardiometabolic conditions such as high BMI, increased waist circumference, hypertension and elevated glucose levels had lower age-standardized levels of free testosterone estimated using both Vermeulen and Zakharov compared to men without these conditions. For cFTV and cFTZ the trends pointed in the same direction irrespective of absolute values or age-standardized values being used. A consistent pattern for cFTV and cFTZ was also seen in relation to long-term outcomes such as type 2 diabetes and CVD mortality with higher levels of free testosterone, irrespective of algorithm, being associated with lower risk of the two outcomes.

On the contrary, for FAI a less consistent pattern was seen in relation to cardiometabolic conditions. Only minor differences in FAI levels were seen with different level of BMI indicating a consistent ratio between TT and SHBG across BMI groups. Moreover, for several of the lifestyle parameters, the trends in age-standardized values of FAI were significantly different from absolute values indicating that age-correction has a major influence on FAI. In relation to long-term outcomes, less clear patterns were seen for FAI; similar associations to IHD, CVD and allcause mortality as for CFTV and cFTZ were seen. However, for type 2 diabetes higher levels of FAI was associated with higher risk of type 2 diabetes whereas higher levels of free testosterone estimated by Vermeulen and Zakharov were associated with a lower risk of type 2 diabetes. While FAI is not based on any biophysical characterization, it is still frequently used but our study provides further support that such empiric assessment does not correlate with clinical assessments or provide any mechanistic insight.

In a study by Ho et al. free testosterone was calculated according to four different algorithms including the algorithm suggested by Vermeulen et al., and subsequently compared to FAI. Interestingly, the authors found that FAI tended to overestimate the free testosterone level at lower levels of SHBG irrespective of which equation was used indicating that FAI is a less reliable index of free testosterone (28). In the present study, we observed lower age-standardized levels of TT and SHBG among men with cardiometabolic characteristics compared to men without such conditions in accordance with a number of other studies (19). Thus, the lowered SHBG level in cardiometabolic men could explain the deviating trends seen for FAI compared to the two other algorithms. In line with this, our findings of higher risk of type 2 diabetes with higher quartile of FAI and a tendency of lower risk of type 2 diabetes with higher quartiles of free testosterone estimated using Vermeulen or Zakharov could be explained as secondary to an observed lower risk of type 2 diabetes with higher quartile of SHBG as previously published (27).

In general, it can be debated whether free testosterone alone reflects the biologically relevant fraction of testosterone in accordance with the free hormone hypothesis (2). However, based on data from the European Male Aging Study an association between men with both low total and free testosterone and impaired sexual function like erectile dysfunction and infrequent morning erections was seen whereas no association was seen for men with only low TT and free testosterone within the reference range (29). The importance of free testosterone was furthermore underlined in a case of a 27-year-old man with undetectable SHBG levels due to a mutation in the SHBG gene (30). The man had low TT level but free testosterone levels within the reference range and was characterized by normal gonadal development and spermatogenesis suggesting a limited role of SHBG and a high TT level. However, the man presented a 7-year history of muscle weakness, fatigue and decreased libido indicating potential androgen deficiency despite normal virilization and normal free testosterone. In relation to the cardiometabolic outcomes included in this study, agreement was seen between associations observed for TT and free testosterone calculated according to Zakharov et al. and Vermeulen et al. indicating that both the total and free fraction of testosterone are equally good indicators in relation to these outcomes in men from the general population. However, to determine the biological relevance of total and free testosterone it would be relevant to include more androgen-specific endpoints.

Despite the differences in absolute levels the algorithms suggested by Vermeulen et al. and Zakharov et al. were in general in agreement in relation to the survival outcomes (Fig. 2). However, the algorithm by Zakharov et al. tended to be in closer agreement with the associations seen for total testosterone indicating that the algorithm is more dependent on the levels of total testosterone compared to the algorithm by Vermeulen et al. However, with findings from recent studies on crystal structure and multisite binding of testosterone with albumin $(31,32)$, it is conceivable that simplified assumptions in extant models should be examined in detail. It is unclear why the algorithm by Vermulen et al. was largely independent

This work is licensed under a Creative Commons Attribution-NonCommercial 4.0 International License. ed from Bioscientifica com at 04/26/2023 07:57:59AM 
of both the levels of SHBG, albumin and total testosterone level in contrast to the algorithm by Zakharov.

In the present study, free testosterone was calculated in men from the general population with only a minor proportion of the men being characterized by extreme hormone levels. The agreement between the algorithms may differ in a clinical setting with patients exhibiting extreme values of binding proteins and/or total testosterone levels where a more comprehensive model incorporating dynamic repartitioning of testosterone between SHBG and albumin bound fractions may be superior.

In addition to baseline levels of metabolic parameters, our follow-up study made it possible to also investigatelongterm cardiovascular outcomes. Some limitations should however be considered when interpreting the results. The aim of the study was to compare different algorithms of free testosterone in relation to metabolic parameters. It would, however, have been relevant also to include direct measurements of free testosterone using equilibrium dialysis to directly compare the different algorithms to the gold standard method. Due to lack of serum, this was however not a possibility. Furthermore, no information on symptoms on androgen insufficiency was available. Thus, a comparison of the three free testosterone algorithms in relation to androgen activity was not possible. Likewise, information on other measures of biological activity of testosterone, such as PSA levels or hematocrit levels, were not available. Information on $\mathrm{LH}$, the primary stimulator of testosterone was however available but the calculated ratio between $\mathrm{LH}$ and the algorithms by Vermeulen et al. and Zakharov et al. did not reveal any differences in relation to the included survival outcomes (data not shown). Another limitation in the study is the lack of measurements of albumin levels which is included for calculation of free testosterone, because of limitations of available serum volume. However, as relatively large changes in albumin is needed to significantly affect the calculated free testosterone level we used a fixed average level of albumin in both the algorithm by Vermeulen et al. and Zakharov et al. Both testosterone and SHBG were measured using immunoassays. Measurements of sex steroids by use of immunoassays have been criticized for being less reliable compared to methods based on liquid or gas chromatography tandem mass spectrometry, which today are recommended for analyses of small molecules like steroid hormones $(1,33)$. The main problem of immunoassays relates to their tendency of overestimating concentrations in the lower range but this issue is less significant in higher measurement ranges $(1,34)$.
Thus, this is not a major concern in this study based on adult men from the general population.

Our findings are based on a random sample of men from the general population with a high participation rate. Thus, we believe that our findings are generalizable to similar Caucasian populations in the same age range. Overall, the findings of the present study indicate that free testosterone is a biological marker of different cardiometabolic endpoints. However, different algorithms of free testosterone are differently associated with these endpoints indicating that the algorithms contribute with different information but also limits any general conclusions. In future studies, endpoints that are more directly related to androgen action would be relevant to include in relation to the different algorithms to further elucidate the differences between algorithms. Alternatively, measurements of the bioactive androgen level using an androgen bioassay could improve the knowledge of biologically relevant free testosterone levels (35).

In conclusion, our findings suggest that free testosterone estimated by Vermeulen and Zakharov are more sensitive markers of morbidity compared to FAI despite systematic differences in absolute levels. Consequently, because of the significant differences in absolute levels of free testosterone, specific reference ranges for each method should be provided. It remains however to be elucidated whether free testosterone alone can explain the effects of androgen action.

\section{Supplementary materials}

This is linked to the online version of the paper at https://doi.org/10.1530/ EC-20-0552.

\section{Declaration of interest}

Dr Jasuja reports receiving NIH grant funding, equity interest in FPT, LLC and participation in a patent on a method to calculate free testosterone concentration. These conflicts are overseen by and managed according to policies of the Office of Industry Interaction of the Mass General Brigham Health Care System. The authors have nothing else to disclose.

\section{Funding}

This study was financially supported by a grant from the Vissing Foundation and from the National Institutes of Health.

\section{References}

1 Rosner W, Auchus RJ, Azziz R, Sluss PM \& Raff H. Position statement: utility, limitations, and pitfalls in measuring testosterone: an endocrine society position statement. Journal of Clinical Endocrinology and Metabolism 200792 405-413. (https://doi.org/10.1210/jc.2006-1864)

2 Mendel CM. The free hormone hypothesis: a physiologically based mathematical model. Endocrine Reviews 198910 232-274. (https:// doi.org/10.1210/edrv-10-3-232) 
3 Travison TG, Araujo AB, Kupelian V, O’Donnell AB \& McKinlay JB. The relative contributions of aging, health, and lifestyle factors to serum testosterone decline in men. Journal of Clinical Endocrinology and Metabolism 200792 549-555. (https://doi.org/10.1210/jc.20061859)

4 Wu FCW, Tajar A, Beynon JM, Pye SR, Silman AJ, Finn JD, O'Neill TW, Bartfai G, Casanueva FF, Forti G, et al. Identification of late-onset hypogonadism in middle-aged and elderly men. New England Journal of Medicine 2010363 123-135. (https://doi. org/10.1056/NEJMoa0911101)

5 Antonio L, Wu FCW, O'Neill TW, Pye SR, Ahern TB, Laurent MR, Huhtaniemi IT, Lean MEJ, Keevil BG, Rastrelli G, et al. Low free testosterone is associated with hypogonadal signs and symptoms in men with normal total testosterone. Journal of Clinical Endocrinology and Metabolism 2016101 2647-2657. (https://doi.org/10.1210/ jc.2015-4106)

6 Huang YP, Liu W, Chen SF, Liu YD, Chen B, Deng CH \& Lu MJ. Free testosterone correlated with erectile dysfunction severity among young men with normal total testosterone. International Journal of Impotence Research 201931 132-138. (https://doi.org/10.1038/ s41443-018-0090-y)

7 Ly LP \& Handelsman DJ. Empirical estimation of free testosterone from testosterone and sex hormone-binding globulin immunoassays. European Journal of Endocrinology 2005152 471-478. (https://doi. org/10.1530/eje.1.01844)

8 Keevil BG \& Adaway J. Assessment of free testosterone concentration. Journal of Steroid Biochemistry and Molecular Biology 2019190 207-211. (https://doi.org/10.1016/j.jsbmb.2019.04.008)

9 Woodward MJ, Obsekov V, Jacobson MH, Kahn LG \& Trasande L. Phthalates and sex steroid hormones among men from nhanes, 2013-2016. Journal of Clinical Endocrinology and Metabolism 2020105 E1225-E1234. (https://doi.org/10.1210/clinem/dgaa039)

10 Lerchbaum E, Pilz S, Trummer C, Schwetz V, Pachernegg O, Heijboer AC \& Obermayer-Pietsch B. Vitamin D and testosterone in healthy men: a randomized controlled trial. Journal of Clinical Endocrinology and Metabolism 2017102 4292-4302. (https://doi. org/10.1210/jc.2017-01428)

11 Vermeulen A, Verdonck L \& Kaufman JM. A critical evaluation of simple methods for the estimation of free testosterone in serum. Journal of Clinical Endocrinology and Metabolism 199984 3666-3672. (https://doi.org/10.1210/jcem.84.10.6079)

12 Bhasin S, Brito JP, Cunningham GR, Hayes FJ, Hodis HN, Matsumoto AM, Snyder PJ, Swerdloff RS, Wu FC \& Yialamas MA. Testosterone therapy in men with hypogonadism: an Endocrine Society clinical practice guideline. Journal of Clinical Endocrinology and Metabolism 2018103 1715-1744. (https://doi.org/10.1210/jc.201800229)

13 Avvakumov GV, Grishkovskaya I, Muller YA \& Hammond GL. Resolution of the human sex hormone-binding globulin dimer interface and evidence for two steroid-binding sites per homodimer. Journal of Biological Chemistry 2001276 34453-34457. (https://doi. org/10.1074/jbc.M106274200)

14 Metzger J, Schnitzbauer A, Meyer M, So M, Cuilleron CY, Hauptmann H, Huber E \& Luppa PB. Binding analysis of 1R- and 17R-dihydrotestosterone derivatives to homodimeric sex hormonebinding globulin. Biochemistry 200342 13735-13745. (https://doi. org/10.1021/bi035269k)

15 Olsman N \& Goentoro L. Allosteric proteins as logarithmic sensors. PNAS 2016113 E4423-E4430. (https://doi.org/10.1073/ pnas.1601791113)

16 Motlagh HN, Wrabl JO, Li J \& Hilser VJ. The ensemble nature of allostery. Nature $2014 \mathbf{5 0 8} 331-339$. (https://doi.org/10.1038/ nature13001)

17 Zakharov MN, Bhasin S, Travison TG, Xue R, Ulloor J, Vasan RS, Carter E, Wu F \& Jasuja R. A multi-step, dynamic allosteric model of testosterone's binding to sex hormone binding globulin.
Molecular and Cellular Endocrinology 2015399 190-200. (https://doi. org/10.1016/j.mce.2014.09.001)

18 Fiers T, Wu F, Moghetti P, Vanderschueren D, Lapauw B \& Kaufman JM. Reassessing free-testosterone calculation by liquid chromatography-tandem mass spectrometry direct equilibrium dialysis. Journal of Clinical Endocrinology and Metabolism 2018103 2167-2174. (https://doi.org/10.1210/jc.2017-02360)

19 Brand JS, Rovers MM, Yeap BB, Schneider HJ, Tuomainen TP, Haring R, Corona G, Onat A, Maggio M, Bouchard C, et al. Testosterone, sex hormone-binding globulin and the metabolic syndrome in men: an individual participant data meta-analysis of observational studies. PLOS ONE 20149 e100409. (https://doi. org/10.1371/journal.pone.0100409)

20 Holmboe SA, Vradi E, Jensen TK, Linneberg A, Husemoen LLN, Scheike T, Skakkebæk NE, Juul A \& Andersson AM. The association of reproductive hormone levels and all-cause, cancer, and cardiovascular disease mortality in men. Journal of Clinical Endocrinology and Metabolism 2015100 4472-4480. (https://doi. org/10.1210/jc.2015-2460)

21 Gerdes LU, Brønnum-Hansen H, Madsen M, Borch-Johnsen K, Jørgensen T, Sjøl A \& Schroll M. Trends in selected biological risk factors for cardiovascular diseases in the Danish Monica population, 1982-1992. Journal of Clinical Epidemiology 200053 427-434. (https://doi.org/10.1016/s0895-4356(99)00193-6)

22 Jorgensen T, Borch-Johnsen K, Thomsen TF, Ibsen H, Glumer C \& Pisinger C. A randomized non-pharmacological intervention study for prevention of ischaemic heart disease: baseline results Inter99 (1). European Journal of Cardiovascular Prevention and Rehabilitation 2003 10 377-386. (https://doi.org/10.1097/01.hjr.0000096541.30533.82)

23 Osler M, Linneberg A, Glumer C \& Jorgensen T. The cohorts at the Research Centre for Prevention and Health, formerly 'the Glostrup Population Studies'. International Journal of Epidemiology 201140 602-610. (https://doi.org/10.1093/ije/dyq041)

24 Andersson AM, Jensen TK, Juul A, Petersen JH, Jørgensen T \& Skakkebaek NE. Secular decline in male testosterone and sex hormone binding globulin serum levels in Danish population surveys. Journal of Clinical Endocrinology and Metabolism 200792 4696-4705. (https://doi.org/10.1210/jc.2006-2633)

25 Schmidt M, Pedersen L \& Sørensen HT. The Danish Civil Registration System as a tool in epidemiology. European Journal of Epidemiology 201429 541-549. (https://doi.org/10.1007/s10654-014-9930-3)

26 Helweg-Larsen K. The Danish register of causes of death. Scandinavian Journal of Public Health 201139 (Supplement) 26-29. (https://doi. org/10.1177/1403494811399958)

27 Holmboe SA, Jensen TK, Linneberg A, Scheike T, Thuesen BH, Skakkebaek NE, Juul A \& Andersson AM. Low testosterone: a risk marker rather than a risk factor for type 2 diabetes. Journal of Clinical Endocrinology and Metabolism 2016101 3180-3190. (https://doi. org/10.1210/jc.2016-1778)

28 Ho CKM, Stoddart M, Walton M, Anderson RA \& Beckett GJ. Calculated free testosterone in men: comparison of four equations and with free androgen index. Annals of Clinical Biochemistry $2006 \mathbf{4 3}$ 389-397. (https://doi.org/10.1258/000456306778520115)

29 Rastrelli G, Neill TWO, Ahern T, Bártfai G, Casanueva FF, Forti G, Keevil B, Giwercman A, Han TS, Slowikowska-Hilczer J, et al. Symptomatic androgen deficiency develops only when both total and free testosterone decline in obese men who may have incident biochemical secondary hypogonadism: prospective results from the EMAS. Clinical Endocrinology 201889 459-469. (https://doi. org/10.1111/cen.13756)

30 Vos MJ, Mijnhout GS, Rondeel JMM, Baron W \& Groeneveld PHP. Sex hormone binding globulin deficiency due to a homozygous missense mutation. Journal of Clinical Endocrinology and Metabolism 201499 E1798-E1802. (https://doi.org/10.1210/jc.2014-2055)

31 Czub MP, Venkataramany BS, Majorek KA, Handing KB, Porebski PJ, Beeram SR, Suh K, Woolfork AG, Hage DS, Shabalin IG, et al.

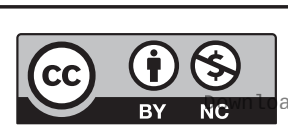

This work is licensed under a Creative Commons Attribution-NonCommercial 4.0 International License. ded from Bioscientifica.com at 04/26/2023 07:57:59AM 
Testosterone meets albumin-the molecular mechanism of sex hormone transport by serum albumins. Chemical Science 201910 1607-1618. (https://doi.org/10.1039/c8sc04397c)

32 Jayaraj A, Schwanz HA, Spencer DJ, Bhasin S, Hamilton JA, Jayaram B, Goldman AL, Krishna M, Krishnan M, Shah A, et al. Allosterically coupled multisite binding of testosterone to human serum albumin. Endocrinology 2021162 1-14. (https://doi.org/10.1210/endocr/bqaa199)

33 Rauh M. Steroid measurement with LC-MS/MS. Application examples in pediatrics. Journal of Steroid Biochemistry and Molecular Biology 2010 121 520-527. (https://doi.org/10.1016/j.jsbmb.2009.12.007)
34 Søeborg T, Frederiksen H, Mouritsen A, Johannsen TH, Main KM, Jørgensen N, Petersen JH, Andersson AM \& Juul A. Sex, age, pubertal development and use of oral contraceptives in relation to serum concentrations of DHEA, DHEAS, $17 \alpha$-hydroxyprogesterone, $\delta 4$-androstenedione, testosterone and their ratios in children, adolescents and young adults. Clinica Chimica Acta 2014437 6-13. (https://doi.org/10.1016/j.cca.2014.06.018)

35 Roy P, Alevizaki M \& Huhtaniemi I. In vitro bioassays for androgens and their diagnostic applications. Human Reproduction Update 2008 14 73-82. (https://doi.org/10.1093/humupd/dmm038)

Received in final form 20 January 2021

Accepted 4 February 2021

Accepted Manuscript published online 6 February 2021
This work is licensed under a Creative Commons Attribution-NonCommercial 4.0 International License. 